\title{
Mode-Stirred Chamber Sample Selection Technique Applied to Antenna Correlation Coefficient
}

\author{
Paul Hallbjörner, ${ }^{1}$ Juan D. Sánchez-Heredia, ${ }^{2}$ and Antonio M. Martínez-González² \\ ${ }^{1}$ Electronics Department, SP Technical Research Institute of Sweden, Box 857, 50115 Borås, Sweden \\ ${ }^{2}$ Departamento de Tecnologías de la Información y Comunicaciones, Universidad Politécnica de Cartagena, 30202 Cartagena, Spain
}

Correspondence should be addressed to Paul Hallbjörner, paul.hallbjorner@sp.se

Received 29 November 2011; Accepted 9 January 2012

Academic Editor: David A. Sanchez-Hernandez

Copyright ( $) 2012$ Paul Hallbjörner et al. This is an open access article distributed under the Creative Commons Attribution License, which permits unrestricted use, distribution, and reproduction in any medium, provided the original work is properly cited.

Mode-stirred chambers provide an excellent tool for antenna characterization. Recent research has focused on making the modestirred chamber technique more versatile. One result of these efforts is the sample selection technique, by which a subset of data with specific properties is extracted from a measured set of raw data. This paper presents how to apply the sample selection technique to measurements of the correlation coefficient in dual antenna measurements, for example, for diversity or MIMO applications. Theoretical background and useful mathematical relations are presented. The application is verified through measurements.

\section{Introduction}

Mode-stirred chambers (MSCs) have since about a decade come to widespread use for antenna characterization and tests of wireless terminals [1-5]. Lately, much research has focused on how to make MSCs more flexible and versatile. One example is the sample selection technique [6-8]. It has been demonstrated in previous work to be applicable to the amplitude distribution of a measured signal. This paper presents an extension of the sample selection technique, in which it is applied to the correlation coefficient between the received signals from two antennas. The fundamental principle of the sample selection technique is the same as in previous work; it is merely applied to another parameter. Applying it to the correlation coefficient improves the usefulness of the sample selection technique in tests of diversity and/or MIMO antennas, where the correlation coefficient is critical. The aim of the presented work is to study the efficiency and accuracy of the proposed technique and demonstrate its practical usefulness.

\section{Sample Selection Applied to Correlation Coefficient}

In an MSC measurement, a sequence of samples is recorded. The sample set has a certain statistical distribution, described by a probability density function (PDF). The correlation coefficient is calculated from dual channel measurements of the signals from a pair of antennas. Dual channel data can be described by a bivariate (2D) PDF, according to $[9,10]$

$$
\begin{aligned}
f\left(x_{1}, x_{2}\right)= & \frac{x_{1} x_{2}}{\left(1-\rho^{2}\right) s_{1}^{2} s_{2}^{2}} \exp \left(-\frac{1}{2\left(1-\rho^{2}\right)} \cdot\left(\frac{x_{1}^{2}}{s_{1}^{2}}+\frac{x_{2}^{2}}{s_{2}^{2}}\right)\right) \\
& \cdot I_{0}\left(\frac{\rho x_{1} x_{2}}{\left(1-\rho^{2}\right) s_{1} s_{2}}\right),
\end{aligned}
$$

where $I_{0}$ is the modified Bessel function of the first kind and of order zero. The parameters $s_{1}$ and $s_{2}$ in (1) define the amplitudes on the two channels, respectively, and $\rho$ is 
the complex correlation coefficient between the data on the two channels, that is, $\rho=\left|\rho_{c}\right|$. There can be an imbalance in the initial data, which can be accounted for in the PDF by letting $s_{1} \neq s_{2}$.

Sample selection is here applied for the purpose of achieving a different correlation coefficient than the one at hand for the whole sequence. This means that for one and the same antenna pair, different scenarios can be extracted in subsets of the initial data, something which can be useful in various performance analyses and tests. The sample selection technique can also be used to change the channel imbalance, but this is also possible by simply weighting the data on one of the channels. In this paper, it is assumed that any imbalance in the initial data should be maintained after sample selection.

For illustration, two examples of PDFs according to (1) are plotted in Figure 1. In each plot, the channels are balanced $\left(s_{1}=s_{2}\right)$, and different values of $\rho$ are applied.

2.1. Accuracy in the Result. The accuracy with which the target correlation coefficient can be achieved is investigated through numerical simulations using Matlab. The initial data consists of $N_{i}$ samples distributed according to the PDF $f_{i}(x)$. From these, $N_{t}$ samples are selected which have a specific target PDF denoted by $f_{t}(x)$. The individual branch distributions are first verified, by generating two Rayleigh distributed sequences of initial data with $N_{i}=50000, s_{1 i}=1$, $s_{2 i}=2$, and $\rho_{i}=0.7$. The selection process is carried out with maintained branch amplitudes in the target data, that is, $s_{1 t}=1$ and $s_{2 t}=2$, and with target correlation $\rho_{t}=$ 0.2. Figures 2(a) and 2(b) show the histogram PDF of the simulated data after sample selection, together with a solid curve marking the theoretical Rayleigh PDF.

Following this, the accuracy of the achieved correlation coefficient is investigated by performing sample selection on 500 realizations and plotting the histogram PDF of the achieved correlation coefficients. This is done for two values of $\rho_{i}(0$ and 0.7$)$ and four values of $\rho_{t}(0.3,0.5,0.7$, and $0.9)$, thus in total eight cases. Each case uses $N_{i}=10000$, $s_{1 i}=1$, and $s_{2 t}=2$. For comparison, in each case, sequences of length $N_{t}$ and with target correlation coefficient $\rho_{t}$ are also generated directly, that is, without any sample selection process. Due to the finite length of the sequences, these will also show some spread in the correlation coefficient. Figures 3(a) and 3(b) show PDFs of the achieved correlation coefficients, with histograms for the sample selection results, and solid lines for the directly generated data. The numbers next to the peaks are the $N_{t}$ values. Apparently, the accuracy depends on $\rho_{i}$ and $\rho_{t}$. Comparing the sample selection results with the directly generated data shows that the selection process itself does not increase the uncertainty by any significant amount, even though a small shift in the distribution is seen in Figure 3(a) for $\rho_{t}=0.3$ and $\rho_{t}=$ 0.9. The small shift, as well as some irregularities in the histograms, can be attributed to fluctuations due to the limited number of samples.

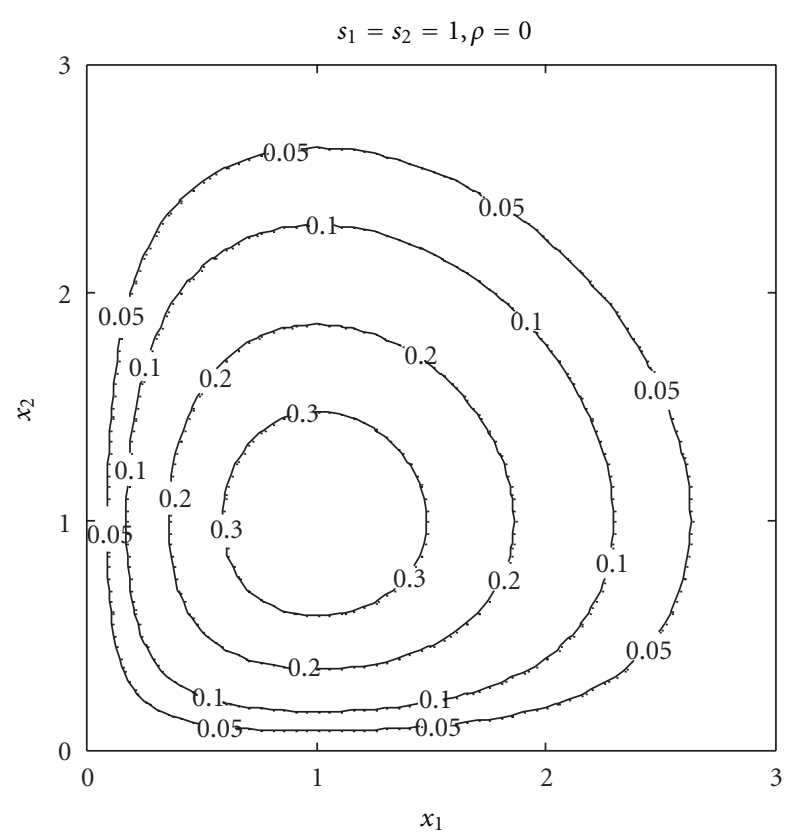

(a)

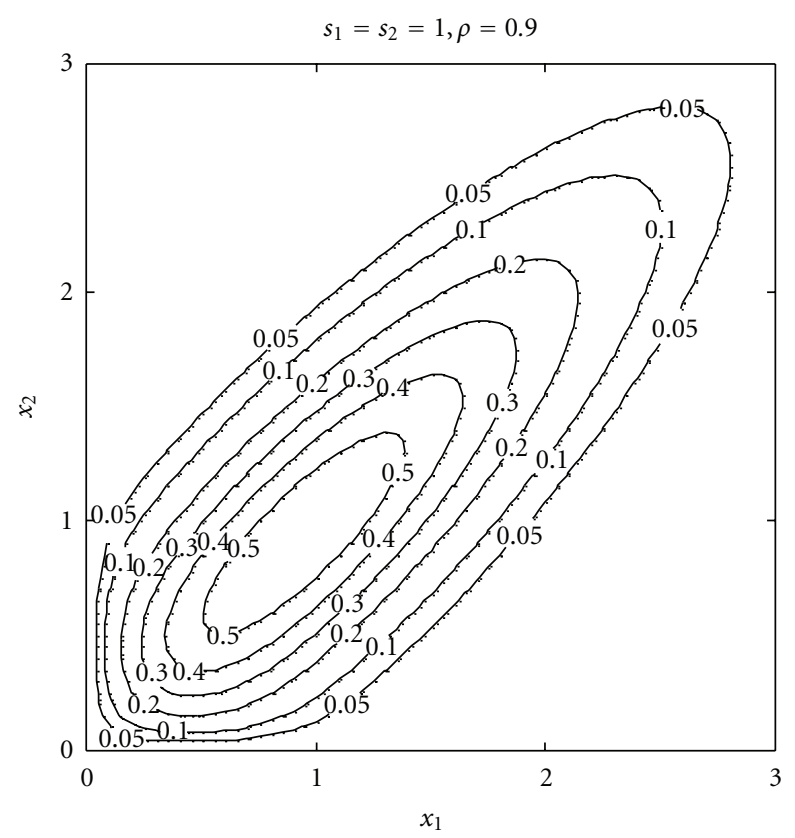

(b)

Figure 1: PDF for 2D Rayleigh distributed data, $\rho=0$ (a) and $\rho=$ 0.9 (b).

Varying $N_{i}$ shows that the spread of the achieved correlation coefficient follows the relation

$$
\sigma_{\rho} \propto \frac{1}{\sqrt{N_{t}}} .
$$

This means that the proportion of remaining samples is critical for the accuracy. 


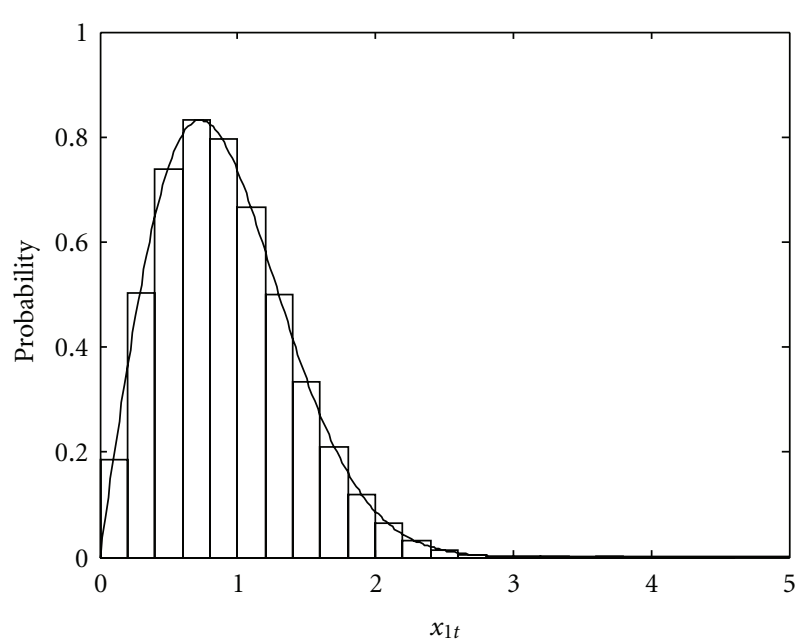

(a)

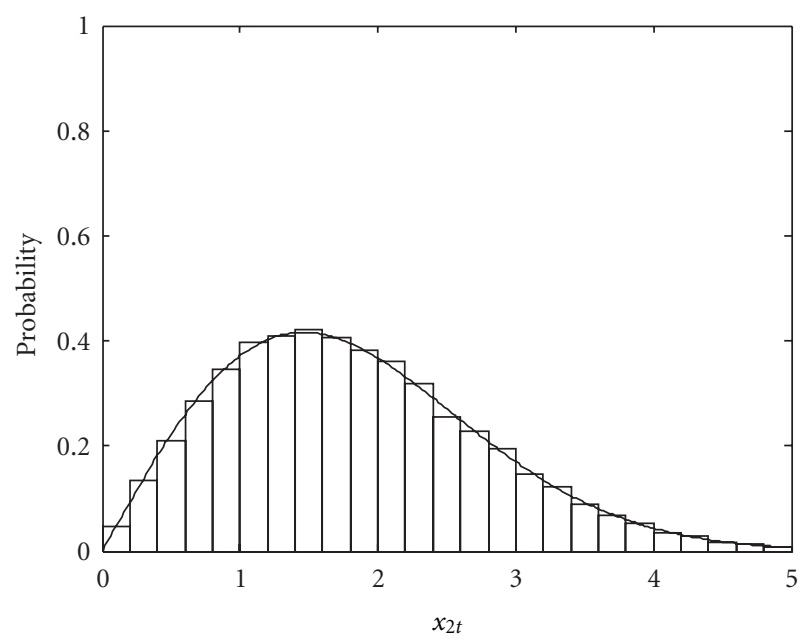

(b)

Figure 2: (a) PDF of the amplitudes on branch 1 of simulated sequence after sample selection, together with theoretical Rayleigh curve for $s=1$. (b) PDF of the amplitudes on branch 2 of simulated sequence after sample selection, together with theoretical Rayleigh curve for $s=2$.

2.2. Proportion of Remaining Samples. The proportion of remaining samples $\left(N_{t} / N_{i}\right)$ in the proposed technique is limited by a theoretical relation [11], which for the general case is

$$
q=\min \left(\frac{f_{i}(x)}{f_{t}(x)}\right)
$$

Substituting (1) in (3), it is found after some manipulations that $q$ is a function of $\rho_{i}$ and $\rho_{t}$, and the expression depends on whether the correlation coefficient is reduced or increased in the sample selection process. For a reduction in correlation coefficient, it is

$$
q_{-}=\frac{1-\rho_{i}^{2}}{1-\rho_{t}^{2}}
$$

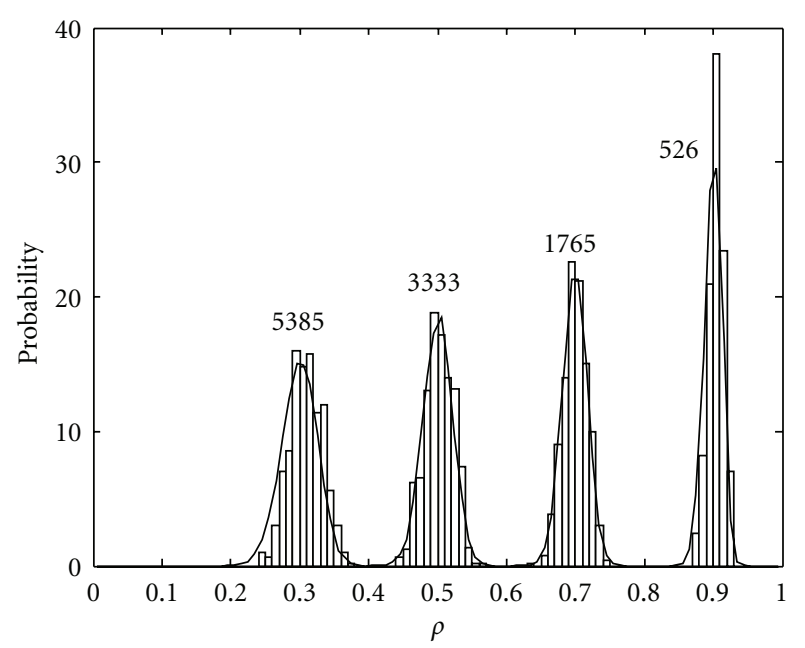

(a)

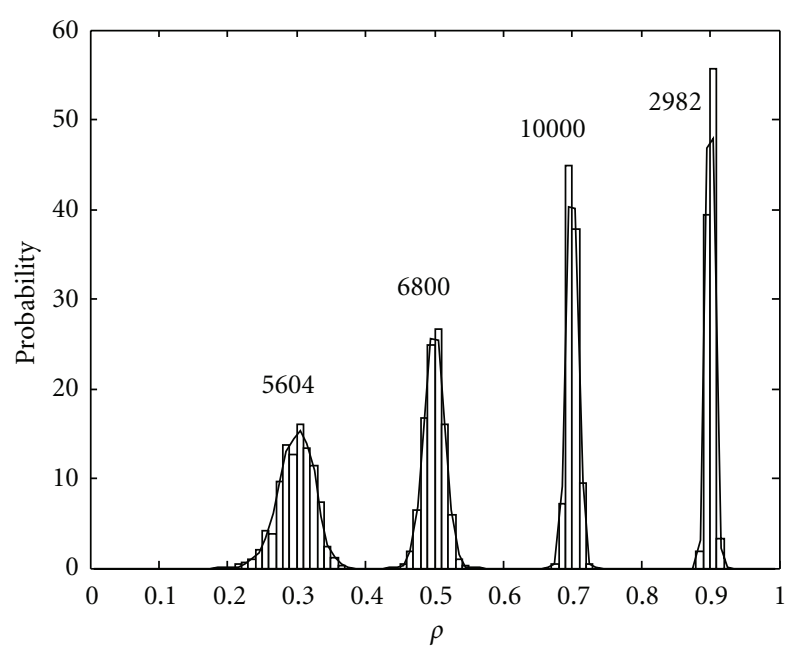

(b)

Figure 3: (a) PDFs for $\rho_{i}=0$ and $\rho_{t}=0.3,0.5,0.7$, and 0.9. $N_{i}=$ 10000 in all cases. Numbers next to the peaks are $N_{t}$ values. Histograms are the results of simulations with 500 realizations. Solid lines show spreads due to finite sequence length for the $N_{t}$ values at hand. (b) PDFs for $\rho_{i}=0.7$ and $\rho_{t}=0.3,0.5,0.7$, and 0.9. $N_{i}=10000$ in all cases. Numbers next to the peaks are $N_{t}$ values. Histograms are the results of simulations with 500 realizations. Solid lines show spreads due to finite sequence length for the $N_{t}$ values at hand.

whereas for an increase in correlation coefficient, it is

$$
q_{+}=\frac{\left(1+\rho_{i}\right)\left(1-\rho_{t}\right)}{\left(1-\rho_{i}\right)\left(1+\rho_{t}\right)} .
$$

Equations (4) and (5) are illustrated in Figure 4, as functions of $\rho_{t}$ for three different values of $\rho_{i}$.

\section{Measurement Example}

Measurements are performed in a mode-stirred chamber, on a pair of mobile terminal antennas. The measurement frequency is $2600 \mathrm{MHz}$. The data is recorded with a network 


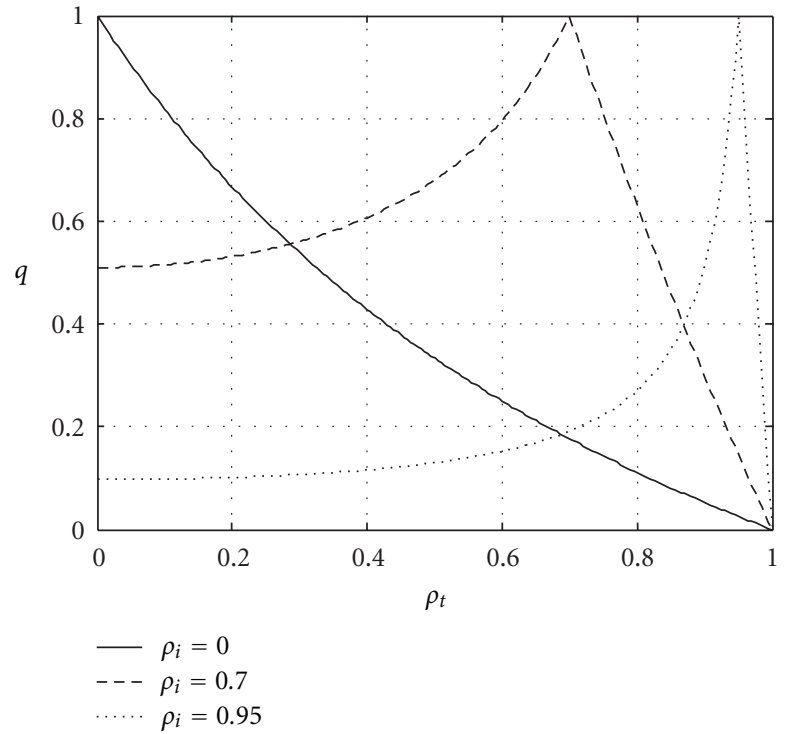

Figure 4: Proportion of remaining samples as a function of target correlation coefficient $\rho_{t}$, for three different values of initial correlation coefficient $\rho_{i}$.

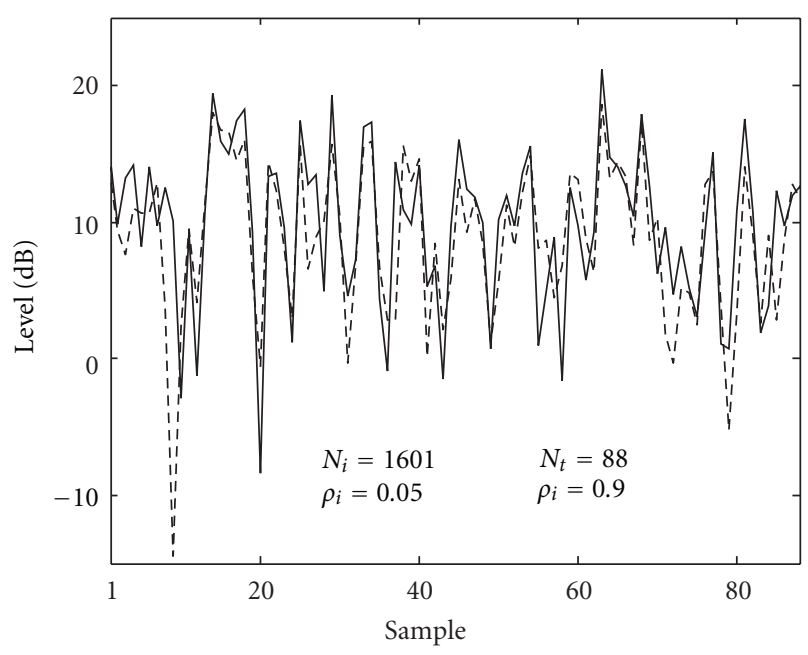

FIGURE 5: Remaining sequences after selection, with $\rho_{t}=0.9$. Solid line is channel 1 and broken line is channel 2 .

analyzer, as two simultaneous sequences of transmission coefficients, each of 1601 samples in length. These sequences constitute the initial data. A small imbalance of $1 \mathrm{~dB}$ (amplitude ratio 1.13) and a correlation coefficient of 0.05 are found in the initial data.

The sample selection technique is then applied for the purpose of increasing the correlation coefficient to 0.9 , while maintaining the same imbalance. According to (5), $q=$ 0.058 , which means that it should be possible to have 93 samples in the output sequences. In practice, for relatively short sequences such as these, different realizations will give slightly different values of $N_{t}$ due to statistical fluctuations. Results of two realizations are presented in Figures 5 and 6. Figure 5 shows the resulting sequences on the two channels,

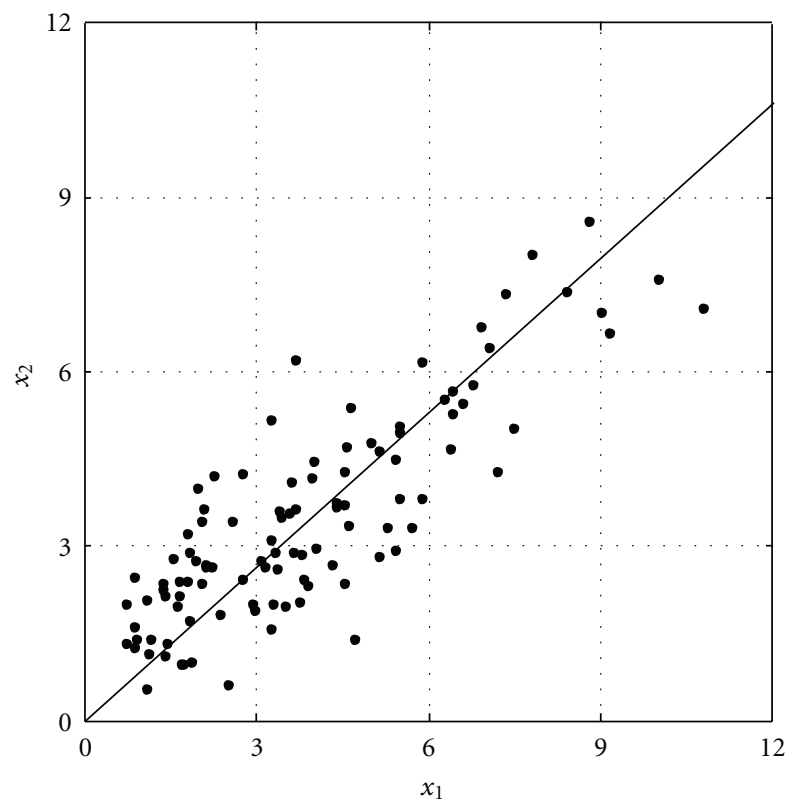

FIgURE 6: Remaining samples after selection, with $\rho_{t}=0.9$.

with $N_{t}=88$ and $\rho_{t}=0.9$ achieved. Figure 6 shows the sample vectors, that is, $\left(x_{1}, x_{2}\right)$ pairs, of a resulting sequence. These figures show that the vectors are correlated and that the imbalance, marked with a solid line in Figure 6, is maintained after sample selection.

\section{Conclusion}

It is demonstrated how the sample selection technique can be applied to the correlation coefficient between the signals from a pair of antennas. The initial sequences can have any correlation coefficient in the interval $(0,1)$, as well as any mean power imbalance. The sample selection technique can achieve any correlation coefficient within the interval $(0,1)$ in the result. Simulations show that using sample selection does not reduce the accuracy in the result, compared to data generated directly with the desired correlation coefficient. Expressions for the maximum proportion of remaining samples are presented. The proposed technique is demonstrated on measured dual channel data from a modestirred chamber.

\section{Acknowledgments}

This work was supported in part by the Spanish National R \& D Programme through TEC 2008-05811 and by Fundación Séneca, the R \& D coordinating agency for the Region of Murcia (Spain) under the 11783/PI/09 and 11610/IV2/09 Projects.

\section{References}

[1] H. A. Mendes, "A new approach to electromagnetic fieldstrength measurements in shielded enclosures," Wescon, Los Angeles, Calif, USA, August 1968. 
[2] P. Corona, G. Latmiral, E. Paolini, and L. Piccioli, "Use of a reverberating enclosure for measurements of radiated power in the microwave range," IEEE Transactions on Electromagnetic Compatibility, vol. 18, no. 2, pp. 54-59, 1976.

[3] J. Krogerus, Comparison of methods for measuring total radiated power and radiation efficiency of handset antennas, M.S. thesis, Master of Science Thesis at Helsinki University of Technology, Department of Electrical and Communications Engineering, 1999.

[4] K. Madsén and P. Hallbjörner, "Reverberation chamber for mobile phone antenna tests," in Proceedings of the Reverberation Chamber, Anechoic Chamber and OATS Users Meeting, Seattle, Wash, USA, June 2001.

[5] J. F. Valenzuela-Valdês, A. M. Martínez-González, and D. A. Sánchez-Hernández, "Emulation of MIMO nonisotropic fading environments with reverberation chambers," IEEE Antennas and Wireless Propagation Letters, vol. 7, Article ID 928488, pp. 325-328, 2008.

[6] EMITE Ing. Document R4-101436, "Standardized fading channel emulation for MIMO OTA using a mode-stirred chamber with sample selection method," in Proceedings of the 3GPP TSG RAN WG4 Adhoc\#2 Meeting, Dublin, Ireland, April 2010.

[7] J. D. Sánchez-Heredia, M. Grudén, J. F. Valenzuela-Valdés, and D. A. Sanchez-Hernandez, "Sample-selection method for arbitrary fading emulation using mode-stirred chambers," IEEE Antennas and Wireless Propagation Letters, vol. 9, Article ID 5460965, pp. 409-412, 2010.

[8] J. D. Sánchez Heredia, M. A. Garcia Fernandez, M. Grudén et al., "Arbitrary fading emulation using mode-stirred reverberation chambers with stochastic sample handling," in Proceedings of the 5th European Conference on Antennas and Propagation (EuCAP '11), pp. 152-154, Rome, Italy, April 2011.

[9] M. Nakagami, "The m-distribution-a general formula of intensity distribution of rapid fading," in Statistical Methods in Radio Wave Propagation, W.C. Hoffman, Pergamon, 1960.

[10] C. Tellambura and A. D. S. Jayalath, "Generation of bivariate rayleigh and nakagami-m fading envelopes," IEEE Communications Letters, vol. 4, no. 5, pp. 170-172, 2000.

[11] P. Hallbjörner, J. D. Sánchez-Heredia, E. de los Reyes, and D. A. Sánchez-Hernández, "Limit for the proportion of remaining samples in the mode-stirred chamber sample selection technique," Microwave and Optical Technology Letters, vol. 53, no. 11, pp. 2608-2610, 2011. 

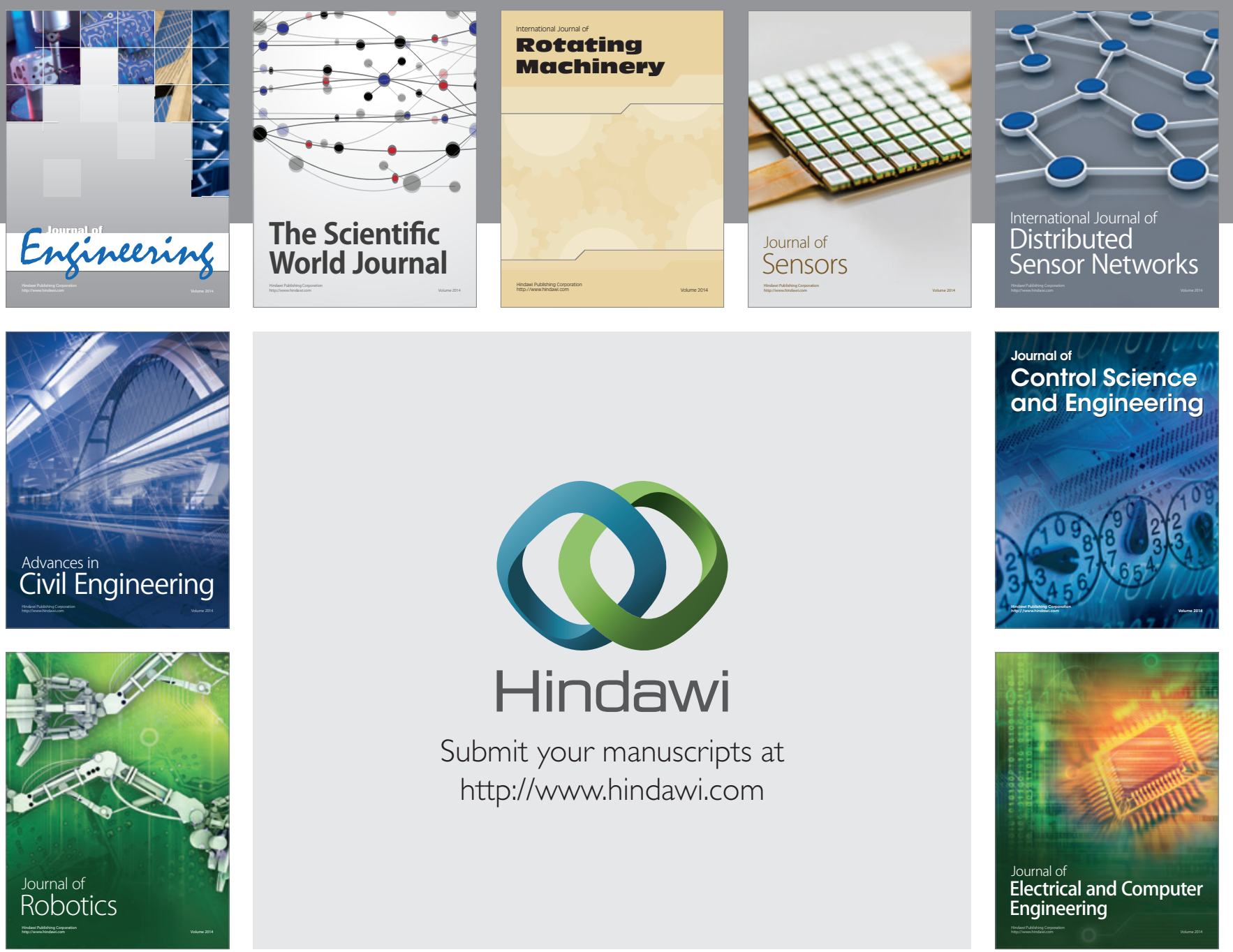

Submit your manuscripts at

http://www.hindawi.com
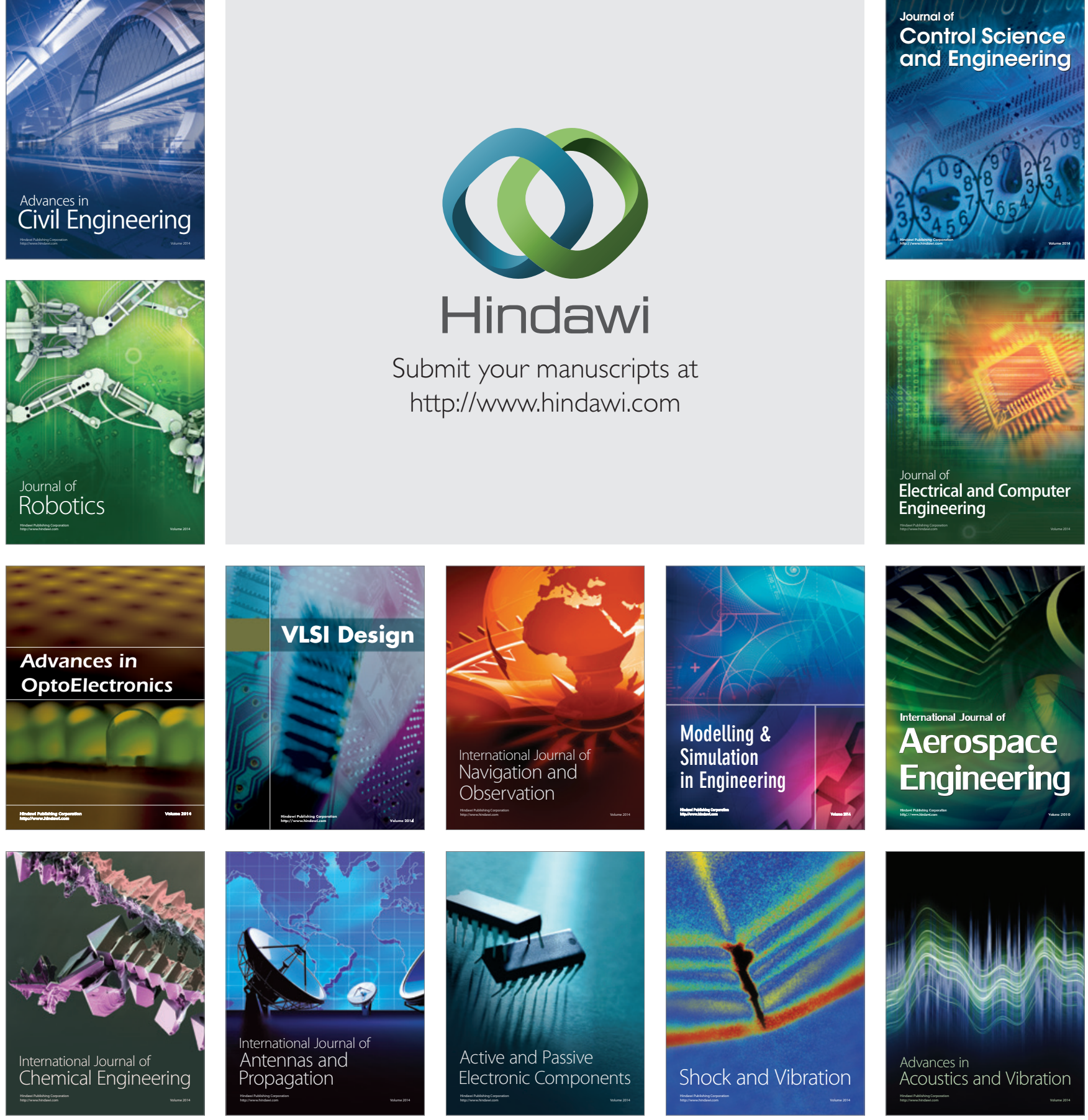